\title{
Fenologia reprodutiva e vegetativa de arbustos endêmicos de campo rupestre na Serra do Cipó, Sudeste do Brasil
}

Reproductive and vegetative phenology of endemic shrubs from Serra do Cipó rupestrian grasslands, Southeastern Brazil

\author{
Renata M. Belo ${ }^{1}$, Daniel Negreiros ${ }^{2,5}$, G. Wilson Fernandes ${ }^{2}$, Fernando A.O. Silveira ${ }^{3}$, \\ Bernardo D. Ranieri ${ }^{2}$ \& Patrícia C. Morellato ${ }^{4}$
}

\begin{abstract}
Resumo
Os Campos rupestres têm destaque no cenário mundial da conservação por sua enorme riqueza em espécies e alta taxa de endemismo. É considerado um ecossistema ameaçado devido à intensa e progressiva descaracterização que vêm sofrendo pela ação antrópica. O objetivo deste estudo foi descrever os padrões fenológicos reprodutivos e vegetativos em seis espécies arbustivas endêmicas dos campos rupestres da Cadeia do Espinhaço, simpátricas na Serra do Cipó, Minas Gerais, e testar a relação entre suas fenofases e a estacionalidade climática. Esperamos que as espécies tenham suas fenofases fortemente relacionadas às variações entre as estações seca e úmida. As observações fenológicas foram conduzidas mensalmente nas fenofases reprodutivas (flor, fruto e dispersão) e vegetativas (queda de folhas e brotamento). De acordo com a combinação dos padrões fenológicos reprodutivos, vegetativos e sazonalidade, foi possivel distinguir quatro estratégias fenológicas para as seis espécies avaliadas. Dessa forma, o presente estudo mostrou uma grande diversidade de padrões fenológicos, mesmo considerando o pequeno número de espécies amostradas. Por outro lado, em todas as espécies as fenofases reprodutivas apresentaram um padrão significativamente sazonal, com alta concentração de espécies reproduzindo em uma dada estação do ano, sugerindo uma importância destacada da sazonalidade do clima na definição dos padrões fenológicos em campos rupestres.
\end{abstract}

Palavras-chave: Cadeia do Espinhaço, endemismo, floração, sazonalidade, sincronia.

\begin{abstract}
The Brazilian "campos rupestres" (high-altitude grasslands) are very important on the world conservation scenario because of high species richness and endemism. These grasslands are regarded as threatened ecosystems due to intense, on-going disruption by man's activities. The aim of this study was to describe the reproductive and vegetative phenological patterns of six shrub species endemic to these grasslands in the Espinhaço Range, sympatric in Serra do Cipó, MG. We tested the relationship between species phenophases and local climate seasonality. We expect that the species phenophases are strongly correlated with variations of the dry and wet seasons. Observations were conducted monthly on reproductive (flowering, fruit production and dispersal) and vegetative (leaf fall and budding) phenophases. Given the combination of reproductive phenology, vegetative phenology, and seasonality, we observed four phenological strategies for the six species. Therefore this study revealed great diversity in phenological patterns, even when considering the small number of species sampled. Moreover, all species showed a significant seasonal pattern for the reproductive phenophases, with high concentrations of species reproducing during a given season, suggesting a key role of climate in defining phenological patterns in the "campo rupestre" grasslands.
\end{abstract}

Key words: Espinhaço mountain range, endemism, flowering, seasonality, synchrony.

\footnotetext{
${ }^{1}$ Universidade de São Paulo, Depto. Ecologia, Lab. Ecofisiologia, 05508-900, São Paulo, SP, Brasil.

${ }^{2}$ Universidade Federal de Minas Gerais, Inst. Ciências Biológicas, Depto. Biologia Geral, Ecologia Evolutiva e Biodiversidade, CP 486, 30161-970, Belo Horizonte, MG, Brasil.

${ }^{3}$ Universidade Federal de Minas Gerais, Inst. Ciências Biológicas, Depto. Botânica, 30161-970, Belo Horizonte, MG, Brasil.

${ }^{4}$ Universidade Estadual Paulista - UNESP, Depto. Botânica, Lab. Fenologia, 13506-900, Rio Claro, SP, Brasil.

${ }^{5}$ Autor para correspondência: negreiros.eco@gmail.com
} 


\section{Introdução}

O campo rupestre é uma fitofisionomia caracterizada por um estrato herbáceo entremeado por pequenos arbustos e subarbustos, distribuídos em mosaicos de micro habitats com substratos que podem variar na fertilidade, capacidade de retenção de água, profundidade do solo e granulometria, além das diferenças no relevo e microclima (Giulietti et al. 1997). Embora este ecossistema se destaque no cenário mundial da conservação por sua enorme riqueza em espécies e alta taxa de endemismo, o campo rupestre pode ser considerado um ecossistema ameaçado pela intensa e progressiva descaracterização que vêm sofrendo pela ação antrópica (Menezes \& Giulietti 2000).

Para embasar estratégias de conservação e restauração em ecossistemas ameaçados, é fundamental promover a investigação dos aspectos básicos da biologia de suas espécies. $\mathrm{O}$ estudo da fenologia representa uma ótima ferramenta para o entendimento dos fatores que influenciam a reprodução e a sobrevivência das espécies vegetais (Morellato et al. 2000, 2010a) contribuindo para a compreensão da dinâmica e estruturação das comunidades de plantas. Além disso, o entendimento dos ciclos reprodutivos das plantas é de fundamental importância para a conservação e manejo de espécies nativas e ameaçadas (Oliveira 2008). Entretanto, os estudos fenológicos com plantas de campo rupestre são escassos, especialmente em espécies endêmicas ou ameaçadas (Madeira \& Fernandes 1999; Conceição et al. 2007; Coelho \& Machado 2009; Dutra et al. 2009; Miola et al. 2010).

As plantas apresentam estratégias fenológicas relacionadas às características ambientais de seus habitats, ao seu hábito, biologia reprodutiva e modo de dispersão (Batalha \& Mantovani 2001; Tannus et al. 2006; Conceição et al. 2007). A interação entre a fenologia de uma determinada espécie de planta e a variação sazonal no clima é um dos determinantes mais importantes de sua distribuição (Chuine \& Beaubien 2001). Assim, espera-se que, principalmente, as espécies endêmicas apresentem respostas fenológicas adaptadas às peculiaridades do habitat no qual ocorrem.

O objetivo do presente estudo foi descrever os padrões fenológicos reprodutivos e vegetativos em seis espécies arbustivas endêmicas dos campos rupestres da Cadeia do Espinhaço, simpátricas na Serra do Cipó, MG, e testar a relação entre suas fenofases e a estacionalidade climática.

\section{Material e Métodos}

Área de estudo e espécies avaliadas

Este estudo foi realizado na Reserva Particular Natural Vellozia (19 $17^{\circ} 46^{\prime \prime} \mathrm{S}, 43^{\circ} 35^{\prime} 28^{\prime \prime} \mathrm{W}$, altitude em torno de $1200 \mathrm{~m}$ ), localizada na Serra do Cipó, porção sul da Cadeia do Espinhaço, Minas Gerais. O clima regional é considerado Cwb na classificação Köppen, com verões quentes e chuvosos e invernos secos e frios, com uma precipitação média anual de 1500 mm (Galvão \& Nimer 1965). Com base na classificação de Madeira \& Fernandes (1999) foram consideradas duas diferentes estações: chuvosa (outubro a abril) e seca (maio a setembro) (Fig. 1). Dados climáticos foram obtidos para o período de coleta dos dados fenológicos, de estação localizada a 4,5 km da área de estudos, no município de Cardeal Mota, Rodovia MG 010, km 98,7, a uma altitude de $860 \mathrm{~m}$ (dados não publicados).

Foram estudadas seis espécies simpátricas (cinco arbustos e uma palmeira com porte arbustivo) na Serra do Cipó: Syagrus glaucescens Glaz. ex Becc. (Arecaceae) é uma pequena palmeira endêmica dos campos rupestres, associada a afloramentos rochosos, que pode atingir até $4 \mathrm{~m}$ de altura (Pintaud et al. 2008; Miola et al. 2010; 2011); Mimosa foliolosa Benth. ssp. pachycarpa (Bentham) (Fabaceae) é um arbusto endêmico das elevações da Cadeia do Espinhaço, ocorre em
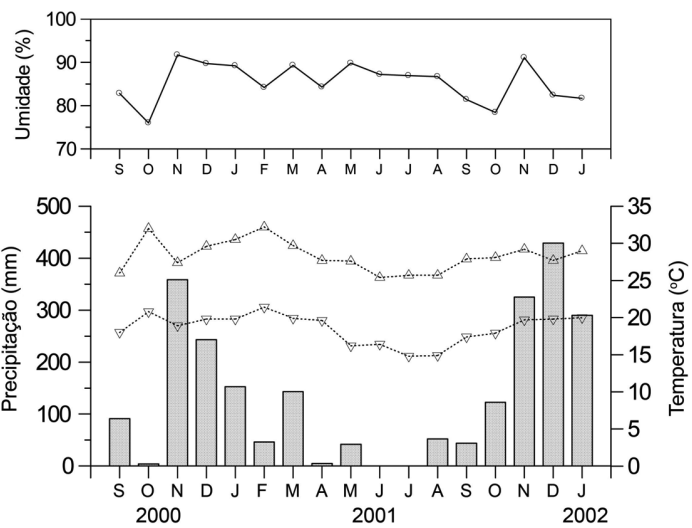

Figura 1 - Variação média mensal na umidade relativa do ar (\%, linha contínua) e temperaturas máxima e mínima ( ${ }^{\circ} \mathrm{C}$, linhas pontilhadas) e precipitação mensal total (mm, barras) durante o período de estudo (2000 a 2002), na Serra do Cipó, MG, Brasil.

Figure 1 - Mean monthly variations in Relative air humidity (\%, continuous line) and maximum and minimum temperatures $\left({ }^{\circ} \mathrm{C}\right.$, dashed lines) and total precipitation ( $\mathrm{mm}$, bars) during the period of study (2000 to 2002), Serra do Cipó, MG, Brazil. 
solos arenosos de campo rupestre e nas bordas de afloramentos de arenito e atinge de 0,4 a 0,7 m de altura (Barneby 1991; Negreiros et al. 2009); Diplusodon orbicularis Koehne (Lythraceae) é um arbusto endêmico da Serra do Cipó, associado a solos arenosos e areno-pedregosos, atingindo de 0,15 a $2 \mathrm{~m}$ de altura (Cavalcanti 1990, Silveira et al. 2012); Comolia sertularia Triana (Melastomataceae: Melastomeae) é um arbusto endêmico da Serra de São José e Serra do Cipó (Drummond et al. 2007), geralmente associado a afloramentos rochosos, atingindo aproximadamente $1 \mathrm{~m}$ de altura; Microlicia tetrasticha Cogn. (Melastomataceae: Microliceae) é um arbusto endêmico da Cadeia do Espinhaço (Cogniaux 1883-1885), crescendo em campos arenosos úmidos (Martins et al. 2009), atingindo aproximadamente 0,5 m de altura; Coccoloba cereifera Schwacke (Polygonaceae) é um arbusto endêmico restrito a uma área de $26 \mathrm{~km}^{2}$ na Serra do Cipó (Melo 2000; Silva et al. 2008; Moreira et al. 2008, 2010), está associado a solos arenosos em vegetação aberta e atinge $2 \mathrm{~m}$ de altura (Ribeiro \& Fernandes 2000).

\section{Coleta e análise de dados}

Ao longo de 12 meses, foram coletados os dados referentes à fenologia qualitativa de 30 indivíduos de cada espécie aleatoriamente selecionados na área de estudo. As observações fenológicas foram feitas mensalmente entre outubro/2000 e setembro/2001 para C. cereifera, $S$. glaucescens e D. orbicularis; entre setembro/2000 e agosto/2001 para $M$. foliolosa e entre janeiro/2001 e dezembro/2001 para $C$. sertularia e $M$. tetrasticha. Em cada indivíduo foi registrada a presença ou ausência de: botões e/ou flores, indiscriminadamente (floração); frutos em fase de maturação (frutificação) (Tannus et al. 2006); frutos em dispersão, considerando frutos abertos e expostos à ação do vento, gravidade ou de dispersores animais (dispersão); brotos e/ou folhas novas (brotamento); e queda foliar (senescência) (veja Morellato et al. 1989).

Os padrões fenológicos das espécies foram classificados em contínuo ou anual sazonal de acordo com a frequência de indivíduos em cada fenofase ao longo do ano. Foi considerado padrão contínuo a ocorrência de determinada fenofase ao longo do ano todo, com frequência similar e sem interrupções. O padrão anual sazonal (modificado de Newstrom et al. 1994) foi definido pela ocorrência de um evento fenológico durante o ano, com obrigatoriamente um período de maior frequência de indivíduos e com ausência da fenofase por período igual ou maior que dois meses.

Devido à natureza dos dados, análises estatísticas circulares foram utilizadas para detectar o comportamento sazonal em cada espécie, conforme descrito em Morellato et al. (1989, 2000, 2010b) e Zar (1996), utilizando do software Oriana versão 3.0 (Kovach 2003). A frequência de ocorrência de cada evento fenológico para cada espécie foi calculada como em Morellato et al. (1989), convertida em ângulos (360 representam os 365 dias do ano, sendo $1^{\circ}$ de janeiro o ângulo zero), calculada a data ou ângulo médio de ocorrência. O teste de Rayleigh (Zar 1996) foi aplicado para testar a significância do ângulo ou data média das fenofases com distribuição unimodal e se existe um padrão significativamente sazonal (Morellato et al. 2000; Morellato 1989, 2010b). Quando o ângulo médio é significativo o padrão é considerado sazonal, e este corresponde à data média do ano ao redor da qual se concentram os eventos fenológicos (Morellato et al. 2000, 2010b). O vetor $r$ varia de 0 a 1 e indica a concentração dos indivíduos ao redor da data média ou grau de sazonalidade da fenofase (Morellato et al. 2000, 2010b).

Com intuito de averiguar se a frequência de indivíduos em uma determinada fenofase responde proporcionalmente à sazonalidade climática, foi calculado o grau de sincronia de cada espécie nas estações seca e chuvosa aplicando o índice de atividade ou a proporção de indivíduos manifestando determinada fenofase na estação seca ou úmida como sugerido por Bencke \& Morellato (2002a, b): assincrônico $(<20 \%$ dos indivíduos manifestando a fenofase); pouco sincrônico/baixa sincronia (20-60\%); ou alta sincronia (>60\%).

\section{Resultados}

De acordo com a combinação dos padrões fenológicos reprodutivos, vegetativos e sazonalidade, este estudo distinguiu quatro estratégias fenológicas para as seis espécies avaliadas: i) floração, frutificação, dispersão anuais e sazonais na estação chuvosa, brotamento sazonal na estação chuvosa e senescência ausente ou não sazonal (M. foliolosa e C. cereifera); ii) floração na estação chuvosa, frutificação entre a estação chuvosa e seca, e dispersão na seca, brotamento e senescência o ano todo ( $C$. sertularia e $M$. tetrasticha); iii) floração na estação seca, frutificação e dispersão na estação chuvosa, 
brotamento durante todo o ano e senescência ausente (S. glaucescens); iv) floração no final da estação chuvosa, frutificação e dispersão na seca, brotamento o ano todo e senescência na estação seca (D. orbicularis).

Syagrus glaucescens apresentou padrão anual para todas as fenofases reprodutivas (Fig. 2a-c). O pico de floração foi significativamente sazonal com data média no mês de maio, início da estação seca (Tab. 1; Fig. 2a). O sincronismo da floração foi baixo em ambas as estações (Tab. 2). O pico de frutificação foi significativamente sazonal com data média em novembro, durante a estação chuvosa (Tab. 1; Fig. 2b), com baixa sincronia na estação chuvosa e assincronia na seca (Tab. 2). O pico de dispersão de sementes foi sazonalmete significativo, com data média em janeiro, auge da estação chuvosa (Tab. 1; Fig. 2c), com assincronia nas duas estações (Tab. 2). O brotamento apresentou padrão contínuo, com distribuição uniforme ao longo do ano (Tab. 1). Nenhum indivíduo da espécie apresentou sinais de senescência durante todo o ano.

Mimosa foliolosa apresentou padrão anual para todas as fenofases reprodutivas (Fig. 3a-c). O pico de floração foi significativamente sazonal com data média no mês de janeiro, auge da estação chuvosa (Tab. 1; Fig. 3a). A floração foi assincrônica entre os indivíduos observados em ambas as estações (Tab. 2). O pico de frutificação foi significativamente sazonal com data média em outubro, início da estação chuvosa (Tab. 1; Fig. 3b). A frutificação apresentou baixa sincronia na estação chuvosa e assincronia na estação seca (Tab. 2). O pico de dispersão foi significativamente sazonal com data média em outubro, na estação chuvosa (Tab. 1; Fig. 3c). A dispersão apresentou baixa sincronia na estação chuvosa e assincronia na estação seca (Tab. 2). O brotamento apresentou
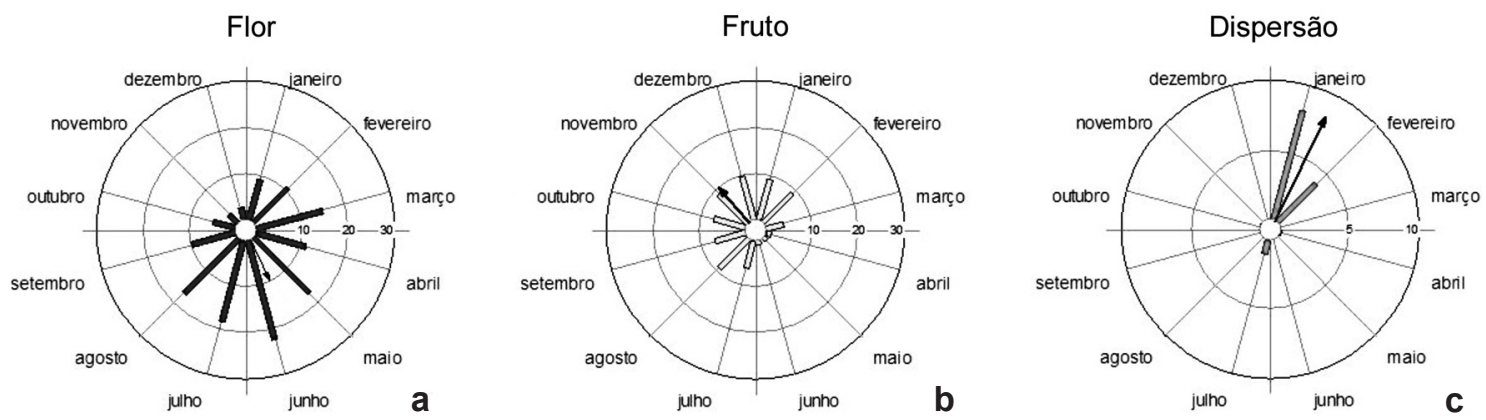

Figura 2 - Diagrama circular do número de indivíduos de Syagrus glaucescens em a. flor; b. fruto; c. dispersão, na Serra do Cipó, MG. A seta aponta para a data média e o comprimento da seta representa o valor de $\mathrm{r}$, que varia de 0 a 1, e mostra a concentração da fenofase ao redor da data média (grau de sazonalidade).

Figure 2 - Circular diagram presenting the number of individuals of Syagrus glauscescens: a. flowering; b. fruiting; c. dispersing, in Serra do Cipó, MG. The arrow points to the mean date, and the length of the arrow represents the value of vector $r$, ranging from 0 to 1 , or the concentration of the phenophase around the mean date (degree of seasonality).
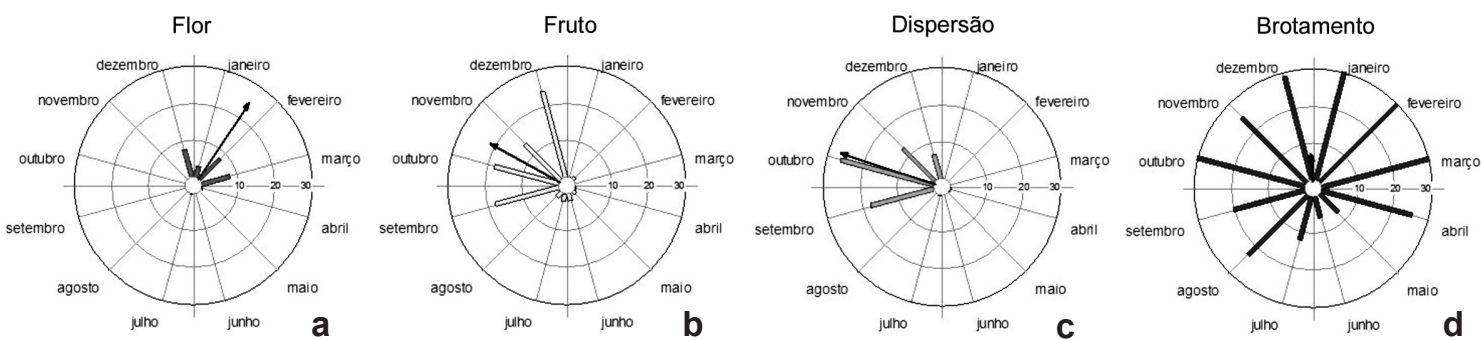

Figura 3 - Diagrama circular do número de indivíduos de Mimosa foliolosa em: a. flor; b. fruto; c. dispersão; d. brotamento, na Serra do Cipó, MG. A seta aponta para a data média e o comprimento da seta representa o valor de r, que varia de 0 a 1 , e mostra a concentração da fenofase ao redor da data média (grau de sazonalidade).

Figure 3 - Circular diagram presenting the number of individuals of Mimosa foliolosa: a. flowering; b. fruiting; c. dispersing; d. leaf flushing in Serra do Cipó, MG. The arrow points to the mean date, and the length of the arrow represents the value of vector $\mathrm{r}$, ranging from 0 to 1 , or the concentration of the phenophase around the mean date (degree of seasonality). 
Tabela 1 - Resultados da análise estatística circular para a ocorrência de sazonalidade nas fenofases de seis espécies endêmicas dos campos rupestres, Serra do Cipó, MG. * = somatório do número total de observações ao longo do ano. $n s=$ diferença não significativa $(p>0,05) ;-=$ não aplicável devido a distribuição uniforme ou baixo número de observações.

Table 1 - Circular statistic analyses for the occurrence of seasonality of each phenophase for six plant species endemic from rupestrian grasslands, Serra do Cipó, MG. * = Total number of observations during the year; $n s=$ a non significant difference $(p>0,05) ;-=$ test not applied due to uniform distribution or low number of observations.

\begin{tabular}{|c|c|c|c|c|c|c|c|}
\hline Espécie & Fenofase & $\begin{array}{l}\text { Número de } \\
\text { observações* }\end{array}$ & $\begin{array}{l}\text { Data } \\
\text { média } \\
\text { (mês) }\end{array}$ & $\begin{array}{l}\text { Ângulo } \\
\text { médio do } \\
\text { vetor }\end{array}$ & $\begin{array}{l}\text { Desvio } \\
\text { padrão } \\
\text { circular }\end{array}$ & $\begin{array}{l}\text { Comprimento } \\
\text { médio do vetor } \\
\mathrm{r}\end{array}$ & $\begin{array}{c}\text { Teste de } \\
\text { uniformidade } \\
\text { Rayleigh }(p)\end{array}$ \\
\hline \multirow{5}{*}{ 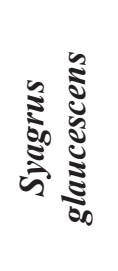 } & Floração & 141 & Maio & $155,52^{\circ}$ & $85,70^{\circ}$ & 0,33 & $<0,001$ \\
\hline & Frutificação & 73 & Novembro & $318,58^{\circ}$ & $84,96^{\circ}$ & 0,33 & $<0,001$ \\
\hline & Dispersão & 13 & Janeiro & $25,82^{\circ}$ & $35,10^{\circ}$ & 0,83 & $<0,001$ \\
\hline & Brotamento & 354 & - & $120,00^{\circ}$ & $164,09^{\circ}$ & 0,02 & $n s$ \\
\hline & Senescência & 0 & - & - & - & - & - \\
\hline \multirow{5}{*}{ 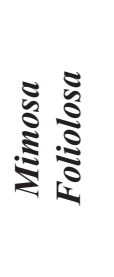 } & Floração & 27 & Janeiro & $33,37^{\circ}$ & $35,73^{\circ}$ & 0,82 & $<0,001$ \\
\hline & Frutificação & 82 & Outubro & $298,65^{\circ}$ & $46,90^{\circ}$ & 0,72 & $<0,001$ \\
\hline & Dispersão & 64 & Outubro & $288,62^{\circ}$ & $27,35^{\circ}$ & 0,90 & $<0,001$ \\
\hline & Brotamento & 267 & Dezembro & $356,03^{\circ}$ & $97,90^{\circ}$ & 0,23 & $<0,001$ \\
\hline & Senescência & 4 & - & - & - & - & - \\
\hline \multirow{5}{*}{ 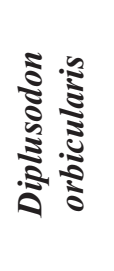 } & Floração & 45 & Abril & $116,94^{\circ}$ & $22,89^{\circ}$ & 0,92 & $<0,001$ \\
\hline & Frutificação & 106 & Julho & $186,86^{\circ}$ & $47,97^{\circ}$ & 0,70 & $<0,001$ \\
\hline & Dispersão & 73 & Setembro & $251,48^{\circ}$ & $33,62^{\circ}$ & 0,84 & $<0,001$ \\
\hline & Brotamento & 269 & - & $9,50^{\circ}$ & $136,97^{\circ}$ & 0,06 & $n s$ \\
\hline & Senescência & 18 & Novembro & $313,24^{\circ}$ & $24,29^{\circ}$ & 0,91 & $<0,001$ \\
\hline \multirow{5}{*}{ 音 } & Floração & 113 & Janeiro & $11,10^{\circ}$ & $65,39^{\circ}$ & 0,52 & $<0,001$ \\
\hline & Frutificação & 197 & Abril & $94,58^{\circ}$ & $86,17^{\circ}$ & 0,32 & $<0,001$ \\
\hline & Dispersão & 112 & Julho & $192,66^{\circ}$ & $62,48^{\circ}$ & 0,55 & $<0,001$ \\
\hline & Brotamento & 351 & - & $45,00^{\circ}$ & $178,34^{\circ}$ & 0,01 & $n s$ \\
\hline & Senescência & 9 & - & $225,00^{\circ}$ & $88,05^{\circ}$ & 0,31 & $n s$ \\
\hline \multirow{5}{*}{ 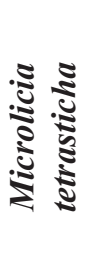 } & Floração & 75 & Dezembro & $344,35^{\circ}$ & $23,89^{\circ}$ & 0,92 & $<0,001$ \\
\hline & Frutificação & 188 & Abril & $93,88^{\circ}$ & $56,60^{\circ}$ & 0,61 & $<0,001$ \\
\hline & Dispersão & 202 & Julho & $193,09^{\circ}$ & $70,28^{\circ}$ & 0,47 & $<0,001$ \\
\hline & Brotamento & 334 & - & $161,76^{\circ}$ & $131,06^{\circ}$ & 0,07 & $n s$ \\
\hline & Senescência & 4 & - & - & - & - & - \\
\hline \multirow{4}{*}{ 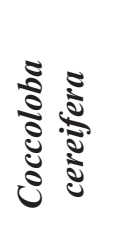 } & Floração & 20 & Novembro & $311,96^{\circ}$ & $73,55^{\circ}$ & 0,44 & $<0,001$ \\
\hline & Frutificação & 20 & Dezembro & $356,53^{\circ}$ & $75,38^{\circ}$ & 0,42 & $<0,001$ \\
\hline & Dispersão & 16 & Janeiro & $29,10^{\circ}$ & $67,25^{\circ}$ & 0,50 & $<0,001$ \\
\hline & Brotamento & 25 & Janeiro & $13,40^{\circ}$ & $102,51^{\circ}$ & 0,20 & $<0,001$ \\
\hline
\end{tabular}


Tabela 2 - Sincronia de fenofases nas estações seca e chuvosa, em porcentagem de indivíduos, para seis espécies endêmicas de campos rupestres, Serra do Cipó, $\mathrm{MG}$. Fl= Floração, Fr= Frutificação, D= Dispersão, B= Brotamento, $\mathrm{S}=$ Senescência. Grau de sincronia: $\mathrm{a}=$ assincrônico, $\mathrm{b}=$ baixa sincronia, $\mathrm{c}=$ alta sincronia (Bencke \& Morellato 2002a). Table 2 - Synchrony of phenophases during the dry and wet seasons in percentage of individuals, for the six endemic species from rupestrian grasslands, Serra do Cipó, $\mathrm{MG}$. FL = Flowering, $\mathrm{Fr}=$ Fruiting, $\mathrm{D}=$ Dispersion, $\mathrm{B}=$ Leaf flushing and $\mathrm{S}=$ Senescence. Degree of synchrony: $\mathrm{a}=$ asynchrony, $\mathrm{b}=$ low synchrony and $\mathrm{c}=$ high synchrony (Bencke \& Morellato 2002a)

\begin{tabular}{lcccccccccccc}
\hline & \multicolumn{1}{c}{ Estação chuvosa } & \multicolumn{5}{c}{ Estação seca } \\
\hline Espécie & $\mathrm{Fl}$ & $\mathrm{Fr}$ & $\mathrm{D}$ & $\mathrm{B}$ & $\mathrm{S}$ & & $\mathrm{Fl}$ & $\mathrm{Fr}$ & $\mathrm{D}$ & $\mathrm{B}$ & $\mathrm{S}$ \\
\hline S. glaucescens & $27,6_{\mathrm{b}}$ & $24,2_{\mathrm{b}}$ & $05,7_{\mathrm{a}}$ & $*$ & $0,0_{\mathrm{a}}$ & & $56,0_{\mathrm{b}}$ & $16,0_{\mathrm{a}}$ & $00,7_{\mathrm{a}}$ & $*$ & $0,0_{\mathrm{a}}$ \\
M. foliolosa & $12,9_{\mathrm{a}}$ & $27,1_{\mathrm{b}}$ & $21,9_{\mathrm{b}}$ & $94,8_{\mathrm{c}}$ & $0,0_{\mathrm{a}}$ & & $0,0_{\mathrm{a}}$ & $16,7_{\mathrm{a}}$ & $12,0_{\mathrm{a}}$ & $52,0_{\mathrm{b}}$ & $2,7_{\mathrm{a}}$ \\
D. orbicularis & $15,5_{\mathrm{a}}$ & $07,7_{\mathrm{a}}$ & $13,7_{\mathrm{a}}$ & $*$ & $0,0_{\mathrm{a}}$ & & $15,8_{\mathrm{a}}$ & $77,5_{\mathrm{c}}$ & $41,7_{\mathrm{b}}$ & $*$ & $0,0_{\mathrm{a}}$ \\
C. sertularia & $32,4_{\mathrm{b}}$ & $58,1_{\mathrm{b}}$ & $11,9_{\mathrm{a}}$ & $*$ & $1,9_{\mathrm{a}}$ & & $12,7_{\mathrm{a}}$ & $50,0_{\mathrm{b}}$ & $58,0_{\mathrm{b}}$ & $*$ & $3,3_{\mathrm{a}}$ \\
M. tetrasticha & $35,7_{\mathrm{b}}$ & $57,1_{\mathrm{b}}$ & $27,1_{\mathrm{b}}$ & $*$ & $1,4_{\mathrm{a}}$ & & $00,0_{\mathrm{a}}$ & $45,3_{\mathrm{b}}$ & $96,7_{\mathrm{c}}$ & $*$ & $0,7_{\mathrm{a}}$ \\
C. cereifera & $35,2_{\mathrm{b}}$ & $44,8_{\mathrm{b}}$ & $27,6_{\mathrm{b}}$ & $69,2_{\mathrm{c}}$ & $0,0_{\mathrm{a}}$ & & $19,3_{\mathrm{a}}$ & $14,0_{\mathrm{a}}$ & $10,0_{\mathrm{a}}$ & $36,7_{\mathrm{b}}$ & $0,0_{\mathrm{a}}$ \\
\hline
\end{tabular}

* Fenofase contínua ao longo do ano, não sazonal, sem variação na sincronia

padrão contínuo, com sazonalidade significativa no mês de dezembro, auge da estação chuvosa (Tab. 1; Fig. 3d). O brotamento mostrou alta sincronia na estação chuvosa e baixa sincronia na seca (Tab. 2). Sinais de senescência foram observados apenas durante a estação seca (padrão anual) para poucos indivíduos, porém a sazonalidade foi não significativa (Tab. 1).

Diplusodon orbicularis apresentou um padrão anual para todas as fenofases reprodutivas (Fig. 4a-c). O pico de floração foi sazonal e significativo com data média no mês de abril, final da estação chuvosa e início da estação seca (Tab. 1; Fig. 4a). A floração foi assincrônica nas duas estações (Tab. 2). O pico da frutificação foi significativamente sazonal com data média no mês de julho, auge da estação seca (Tab. 1; Fig. 4b). A frutificação foi assincrônica na estação chuvosa e com alta sincronia na seca (Tab. 2). O pico de dispersão foi significativamente sazonal com data média em setembro (Tab. 1; Fig. 4c). A dispersão foi assincrônica na estação chuvosa e com baixa sincronia na estação seca (Tab. 2). O brotamento apresentou padrão contínuo, com distribuição uniforme ao longo do ano (Tab. 1), sem sazonalidade. A senescência apresentou padrão anual, com sazonalidade significativa e data média no mês de novembro (Tab. 1, Fig. 4d).

Comolia sertularia apresentou padrão contínuo para todas as fenofases reprodutivas, definido pela presença de indivíduos reproduzindo o ano todo (Fig. 5a-c); entretanto, houve sempre uma época definida com proporção significativamente maior de indivíduos reproduzindo, padrão sazonal (Tab. 1). O pico de floração foi significativamente sazonal com data média no mês de janeiro, auge da estação chuvosa (Tab. 1; Fig. 5a). A floração foi assincrônica na estação seca e teve baixa sincronia na chuvosa (Tab. 2). O pico de frutificação foi significativamente sazonal, com data média em abril, no final da estação chuvosa e início da seca (Tab. 1; Fig. 5b). A frutificação apresentou baixa sincronia nas duas estações (Tab. 2). O pico de dispersão foi sazonal, com data média no mês de julho, no auge da estação seca (Tab. 1; Fig. 5c). A dispersão foi assincrônica na estação chuvosa e mostrou baixa sincronia na seca (Tab. 2). O brotamento apresentou padrão contínuo, com distribuição uniforme ao longo do ano (Tab. 1) e a senescência apresentou padrão anual, observada em poucos indivíduos, sem sazonalidade significativa (Tab. 1).

Microlicia tetrasticha apresentou padrão anual para floração e frutificação (Fig. 6a-b) e contínuo, mas sazonal para dispersão (Fig. 6c). O pico de floração foi significativamente sazonal, em dezembro, na estação chuvosa, (Tab. 1; Fig. 6a). A floração foi assincrônica na estação seca e apresentou baixa sincronia na chuvosa (Tab. 2). $\mathrm{O}$ pico de frutificação apresentou sazonalidade significativa, com data média em abril, no final da estação chuvosa e início da seca (Tab. 1; Fig. 6b). A frutificação mostrou baixa sincronia nas duas estações (Tab. 2). O pico de dispersão foi sazonalmente significativo, no mês de julho, na estação seca (Tab. 1; Fig. 6c). A dispersão apresentou baixa sincronia na estação chuvosa e alta 

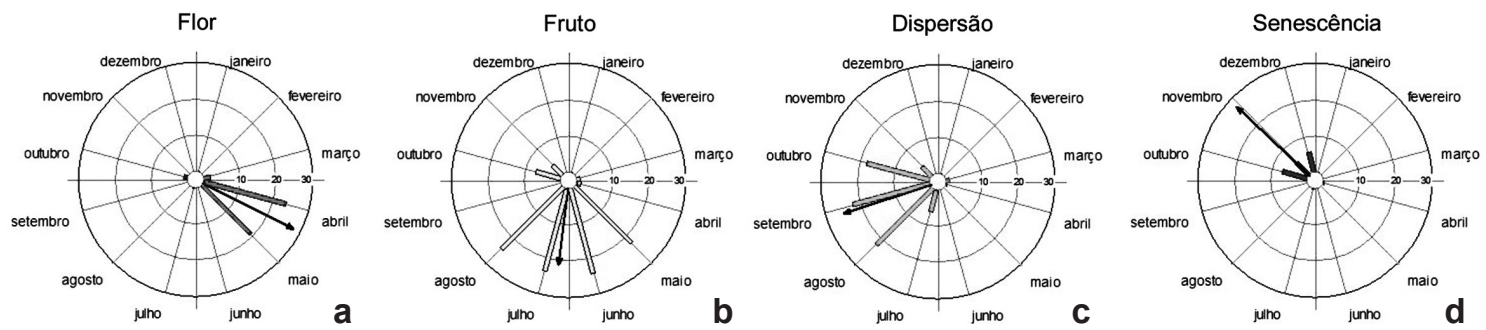

Figura 4 - Diagrama circular do número de indivíduos de Diplusodon orbicularis em: a. flor; b. fruto; c. dispersão; d. senescência, na Serra do Cipó, MG. A seta aponta para a data média e o comprimento da seta representa o valor de r, que varia de 0 a 1 , e mostra a concentração da fenofase ao redor da data média (grau de sazonalidade).

Figure 4 - Circular diagram presenting the number of individuals of Diplusodon orbicularis: a. flowering; b. fruiting; c. dispersing; d. senescence in Serra do Cipó, MG. The arrow points to the mean date, and the length of the arrow represents the value of vector r, ranging from 0 to 1 , or the concentration of the phenophase around the mean date (degree of seasonality).

Flor

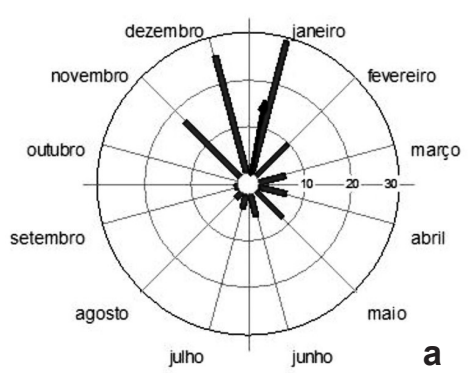

Fruto

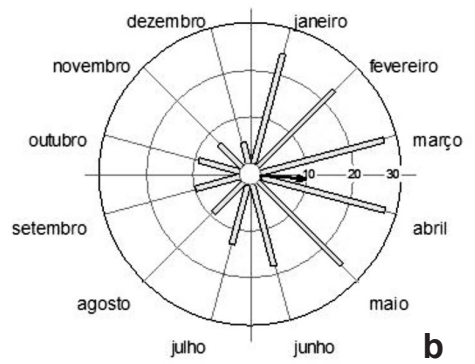

Dispersão

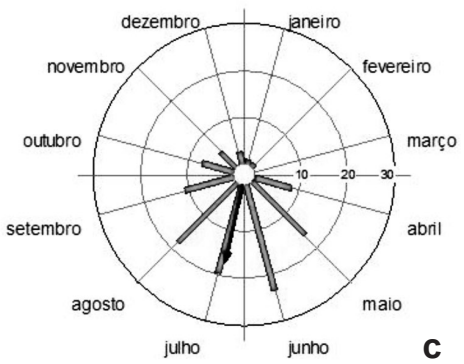

Figura 5 - Diagrama circular do número de indivíduos de Comolia sertularia em: a. flor; b. fruto; c. dispersão, na Serra do Cipó, MG. A seta aponta para a data média e o comprimento da seta representa o valor de r, que varia de 0 a 1, e mostra a concentração da fenofase ao redor da data média (grau de sazonalidade).

Figure 5 - Circular diagram presenting the number of individuals of Comolia sertularia: a. flowering; b. fruiting; c. dispersing, in Serra do Cipó, MG. The arrow points to the mean date, and the length of the arrow represents the value of vector $\mathrm{r}$, ranging from 0 to 1 , or the concentration of the phenophase around the mean date (degree of seasonality).
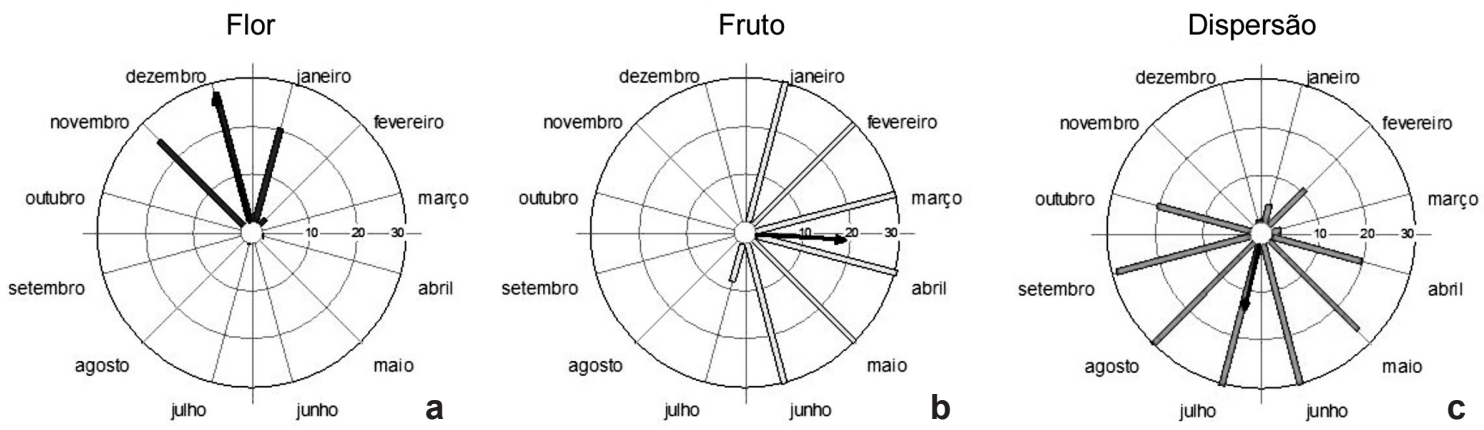

Figura 6 - Diagrama circular do número de indivíduos de Microlicia tetrasticha em: a. flor; b. fruto; c. dispersão, na Serra do Cipó, MG. A seta aponta para a data média e o comprimento da seta representa o valor de r, que varia de 0 a 1 , e mostra a concentração da fenofase ao redor da data média (grau de sazonalidade).

Figure 6 - Circular diagram presenting the number of individuals of Microlicia tetrasticha: a. flowering; b. fruiting; c. dispersing, in Serra do Cipó, MG. The arrow points to the mean date, and the length of the arrow represents the value of vector $\mathrm{r}$, ranging from 0 to 1 , or the concentration of the phenophase around the mean date (degree of seasonality). 

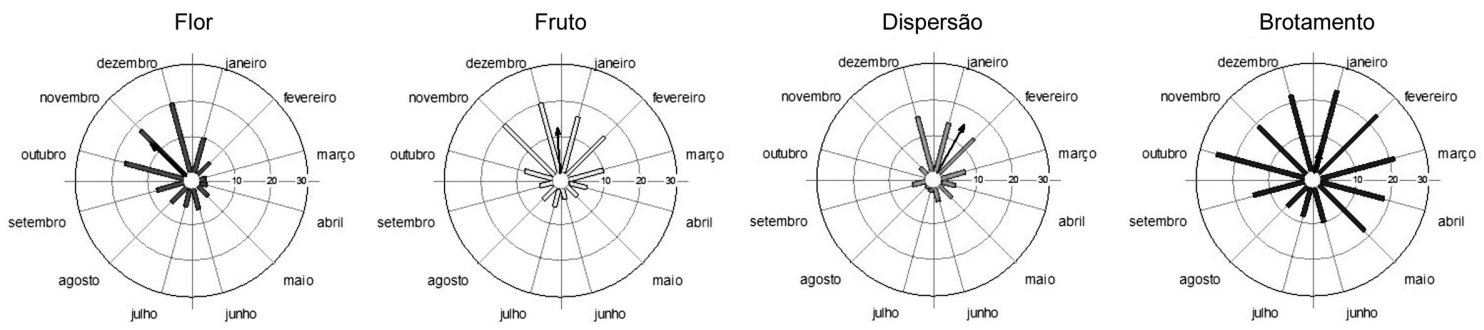

Figura 7 - Diagrama circular do número de indivíduos de Coccoloba cereifera em: a. flor; b. fruto; c. dispersão; d. brotamento, na Serra do Cipó, MG. A seta aponta para a data média e o comprimento da seta representa o valor de r, que varia de 0 a 1, e mostra a concentração da fenofase ao redor da data média (grau de sazonalidade).

Figure 7 - Circular diagram presenting the number of individuals of Coccoloba cereifera: a. flowering; b. fruiting; c. dispersing; d. leaf flushing in Serra do Cipó, MG. The arrow points to the mean date, and the length of the arrow represents the value of vector r, ranging from 0 to 1 , or the concentration of the phenophase around the mean date (degree of seasonality).

sincronia na seca (Tab. 2). O brotamento apresentou padrão contínuo, sem sazonalidade significativa (Tab. 1), enquanto a senescência apresentou padrão anual, com poucos indivíduos na estação seca e sem sazonalidade significativa (Tab. 1).

Coccoloba cereifera apresentou padrão contínuo para todas as fenofases reprodutivas, com picos sazonalmente significativos na estação chuvosa (Fig. 7a-c, Tab. 1). As datas médias para floração, frutificação e dispersão foram respectivamente: novembro, dezembro e janeiro. A floração foi assincrônica na estação seca e pouco sincrônica na chuvosa (Tab. 2). A frutificação e a dispersão apresentaram baixa sincronia dos indivíduos na estação chuvosa e assincronia na estação seca (Tab. 2). O brotamento apresentou padrão contínuo e significativamente sazonal com data média no mês de janeiro, na estação chuvosa (Tab. 1, Fig. 7d), com alta sincronia na estação chuvosa e baixa sincronia na seca (Tab. 2). Não foram observados sinais de senescência nessa espécie durante o período de estudo.

\section{Discussão}

Nosso estudo mostrou uma grande diversidade de padrões fenológicos, mesmo considerando o pequeno número de espécies observadas. A diversidade de padrões fenológicos tem sido relatada para vegetações tropicais como os cerrados e a florestas úmidas (Morellato et al. 2000; Batalha \& Martins 2004; Staggemeier \& Morellato, 2011). Estes estudos consideram que a diversidade fenológica pode ser atribuída tanto a composição em espécies (alta biodiversidade), como ao clima e a heterogeneidade ambiental. Entretanto, para entender a diversidade nesses padrões fenológicos e qual a influencia do clima, ambiente e composição em espécies, é necessário estudar a fenologia de uma ampla amostra das espécies nessa vegetação, ao longo do mosaico vegetacional dos campos rupestres (Staggemeier \& Morellato 2011 e referências neste).

A floração sazonal concentrada na estação seca encontrada para $S$. glaucescens e $D$. orbicularis também foi observada em outras espécies de campo rupestre, como em espécies de Chamaecrista Moench (Fabaceae) na Serra do Cipó (Madeira \& Fernandes, 1999; Costa et al. 2007), duas espécies de Syngonanthus Ruhland (Eriocaulaceae; Cerqueira et al. 2008) e Prepusa montana Mart (Gentianaceae; Coelho \& Machado 2009) na Chapada Diamantina. A floração na época seca pode apresentar vantagens como, por exemplo, a redução da florivoria e a maior atividade dos polinizadores (Janzen 1967; Rathcke \& Lacey 1985), além da diminuição de danos nas estruturas reprodutivas causada pela chuva e eventualmente granizo (Fernandes et al. 2011, 2012).

O padrão de florescimento na estação chuvosa (encontrado para $M$. foliolosa, $C$. cereifera, $C$. sertularia e $M$. tetrasticha) tem sido relatado para a maioria dos arbustos e ervas de áreas tropicais sazonais (Rathke \& Lacey 1985). Este padrão também foi observado em outras espécies de campos rupestres (Dutra et al. 2009), campos sujos (Munhoz \& Felfili 2005; Tannus et al. 2006) e cerrado em geral (Espírito-Santo et al. 2003). O aumento da sincronia de floração entre espécies pode facilitar a atração de polinizadores (Rathke \& Lacey 1985; Piedade-Kiill \& Ranga 2000; Moeller 2004). Assim, o florescimento sincrônico na estação chuvosa pode não só estar 
relacionado à maior disponibilidade hídrica para a formação dos órgãos reprodutivos, como também favorecer a polinização, por potencialmente atrair mais polinizadores.

O período de frutificação está diretamente relacionado às condições que influenciam o sucesso da dispersão e estabelecimento (Rathcke \& Lacey 1985; Morellato et al. 2000; Pedroni et al. 2002). Coccoloba cereifera frutificou no início da estação chuvosa e dispersou em períodos muito próximos, também na estação chuvosa. Segundo observações em espécies zoocóricas dos gêneros Fabaceae e Polygonaceae (Silva et al. 2008), a frutificação e dispersão no período chuvoso é favorecida por manter os frutos mais atrativos aos dispersores por períodos mais longos, propiciando maior sucesso na dispersão (Batalha \& Mantovanni 2001; Batalha \& Martins 2004; Lenza \& Klink 2006).

Diplusodon orbicularis, C. sertularia e $M$. tetrasticha dispersaram sementes na estação seca. Por serem espécies autocóricas, a estação seca favorece a deiscência dos frutos e a dispersão das pequenas sementes, que pode também ser facilitada pela ação dos ventos fortes característicos dos campos rupestres (Frankie et al. 1974; Rathcke \& Lacey 1985).

Todas as espécies apresentaram brotamento ao longo do ano, porém $M$. foliolosa e $C$. cereifera apresentaram redução no número de indivíduos brotando na estação seca. Os sinais de senescência foram fracos e na maioria das espécies avaliadas não ocorreu. Algumas espécies vegetais de savanas tropicais apresentam sistema radicular profundo e eficiente, além da possibilidade da presença de xilopódios, fornecendo condições para que estas espécies consigam se manter sempre verdes durante as estações de seca (Monasterio \& Sarmiento 1976).

Todas as fenofases reprodutivas avaliadas no presente estudo apresentaram um padrão significativamente sazonal, com alta concentração de espécies reproduzindo em uma dada época do ano. Sazonalidade na fenologia reprodutiva também foi observada em outras espécies de campo rupestre (Borba \& Braga 2003; Coelho \& Machado 2009) e em espécies de outras fisionomias abertas como cerrado (Vieira et al. 2002; Batalha \& Martins 2004; Lenza \& Klink 2006) e caatinga (Leite \& Machado 2010), além de fisionomias mais fechadas como florestas tropicais (Pedroni et al. 2002; Lenza \& Oliveira, 2005; Staggemeier \& Morellato 2011). Uma revisão recente da fenologia na América do Sul mostra esta predominância de padrões sazonais para a floração, embora a frutificação possa ser mais variável (Morellato et al. 2013). Entretanto, ainda são poucas as análises de padrões populacionais, embora mostrem um certo grau de sincronia das espécies (e.g., Madeira e Fernandes 1999).

A sincronia entre ciclos reprodutivos e vegetativos e sua ocorrência em estações bem definidas, seca ou úmida, sugere uma importância destacada da sazonalidade do clima na definição dos padrões fenológicos em campos rupestres. Investigações fenológicas mais detalhadas e de longo prazo são fundamentais para confirmar essa relação e possibilitará fazermos previsões sobre o impacto das mudanças climáticas na fenologia das espécies e suas interações nesse ambiente de montanha (e.g., Inouye 2008).

\section{Agradecimentos}

Agradecemos à E. Gressler e M.G.G. Camargo, a leitura prévia do manuscrito, à J.R. Pirani, a identificação das espécies, à T.C. Lana, o auxílio nas coletas de dados, à K.T. Ribeiro por fornecer os dados climatológicos coletados por François Collet. À FAPEMIG (APQ-04105-10), CNPq (163020/2013-2, 482720/2012, 303352/2010-8, 561883/2010-6, 558250/2009-2, 476178/2008-8), CAPES (BEX 323710-9, 02/2009 DRI/CGCI), PELD (403781/2012-4), PPBIO (457519/20126) e FAPEMIG/FAPESP/FAPESPA/VALE S.A (RDP-00048-10), o apoio financeiro em todas etapas da pesquisa. G.W.F. e P.C.M. são bolsistas de produtividade em pesquisa do CNPq. O Laboratório de Fenologia, onde parte das análises e redação foi realizada, recebe apoio da FAPESP.

\section{Referências}

Barneby, R.C. 1991. Sensitivae Censitae. A description of the genus Mimosa Linnaeus (Mimosaceae) in the New World. Memories of New York Botanical Garden 65: 1-835.

Batalha, M.A. \& Mantovani, W. 2001. Reproductive phenological patterns of cerrado plant species at the Pé de Gigante Reserve (Santa Rita do Passa Quatro, SP, Brazil): a comparison between the herbaceous and woody floras. Revista Brasileira de Biologia 60: 129-145.

Batalha, M.A. \& Martins, F.R. 2004. Reproductive phenology of the cerrado plant community in Emas National Park. Australian Journal of Botany 52: 149-161.

Bencke, C.S.C. \& Morellato, L.P.C. 2002a. Comparação de dois métodos de avaliação da fenologia de 
plantas, sua interpretação e representação. Revista Brasileira de Botânica 25: 269-275.

Bencke, C.S.C. \& Morellato, L.P.C. 2002b. Estudo comparativo da fenologia de nove espécies arbóreas em três tipos de floresta atlântica no sudeste do Brasil. Revista Brasileira de Botânica 25: 237-248.

Borba, E.L. \& Braga, P.I.S. 2003. Biologia reprodutiva de Pseudolaelia corcovadensis (Orchidaceae): melitofilia e autocompatibilidade em uma Laeliinae basal. Revista Brasileira de Botânica 26: 541-549.

Cavalcanti, T.C. 1990. Flora da Serra do Cipó, Minas Gerais, Lythraceae. Boletim de Botânica da Universidade de São Paulo 12: 67-93.

Cerqueira, C.O.; Funch, L.S. \& Borba, E.L. 2008. Fenologia de Syngonanthus mucugensis Giul. subsp. mucugensis e S. curralensis Moldenke (Eriocaulaceae), nos municípios de Mucugê e Morro do Chapéu, Chapada Diamantina, BA, Brasil. Acta Botanica Brasilica 22: 962-969.

Chuine, I. \& Beaubien, E.G. 2001. Phenology is a major determinant of tree species range. Ecology Letters 4: 500-510.

Coelho, A.G. \& Machado, C.G. 2009. Fenologia reprodutiva de Prepusa montana Mart. (Gentianaceae) em uma área de campo rupestre da Chapada Diamantina, BA, Brasil. Revista Brasileira de Botânica 32: 405-410.

Cogniaux, A. 1883-1885. Melastomataceae. Tribus Microlicieae e Tibouchinieae. In: Martius, C.F.P. von \& Eichler, A.G. (eds.). Flora brasiliensis. F. Fleischer, Lipsiae, Vol. 14. Pp.5-480.

Conceição, A.A.; Funch, L.S. \& Pirani, J.R. 2007. Reproductive phenology, pollination and seed dispersal syndromes on sandstone outcrop vegetation in the "Chapada Diamantina", northeastern Brazil: population and community analyses. Revista Brasileira de Botânica 30: 475-485.

Costa, C.B.N.; Lambert, S.M.; Borba, E.L. \& Queiroz, L.P. 2007. Post-zygotic reproductive isolation between sympatric taxa in the Chamaecrista desvauxii Complex (Leguminosae-Caesalpinioideae). Annals of Botany 99: 625-635.

Drummond, R.A.R.; Alves, R.J.V. \& Koschnitzke, C. 2007. Melastomataceae da Serra de São José, Minas Gerais. Revista de Biologia Neotropical 4: 1-12.

Dutra, V.F.; Vieira, M.F.; Garcia, F.C.P. \& Lima, H.C. 2009. Fenologia reprodutiva, síndromes de polinização e dispersão em espécies de Leguminosae dos campos rupestres do Parque Estadual do Itacolomi, Minas Gerais, Brasil. Rodriguésia 60: 371-387.

Espírito-Santo, M.M.; Madeira, B.G.; Neves, F.S.; Faria, M.L.; Fagundes, M. \& Fernandes, G.W. 2003. Sexual differences in reproductive phenology and their consequences for the demography of Baccharis dracunculifolia (Asteraceae), a dioecious tropical shrub. Annals of Botany 91:13-19.

Fernandes, G.W.; Oki, Y.; Sanchez-Azofeifa, A.; Faccion, G. \& Amaro-Arruda, H.C. 2011. Hail impact on leaves and endophytes of the endemic threatened Coccoloba cereifera (Polygonaceae). Plant Ecology 212: 1687-1697.

Fernandes, G.W.; Oki, Y.; Sá, C.E.M.; Sales, N.M.; Quintino, A.V.; Mendes, C.F.A. \& Caires, T.B. 2012. Impact of the hailstorm across plant taxa: leaf fall in a mountain environment. Neotropical Biology and Conservation 7: 8-15.

Frankie, G.W.; Baker, H.G. \& Opler, P.A. 1974. Comparative phenological studies of trees in tropical wet and dry forests in the lowlands of Costa Rica. Journal of Ecology 62: 881-913.

Galvão, M.V. \& Nimer, E. 1965. Clima. In: IBGE. (org.). Geografia do Brasil - Grande Região Leste. IBGE, Rio de Janeiro. Pp. 91-139.

Giulietti, A.M.; Pirani, J.R. \& Harley, R.M. 1997. Espinhaço range region, eastern Brazil. In: Davis, S.D.; Heywood, V.H.; Herrera-MacBryde, O.; VillaLobos, J. \& Hamilton, A.C. (eds.). Centres of plant diversity: a guide and strategy for their conservation. Vol.3. WWF/IUCN, Cambridge. Pp. 397-404.

Inouye, D.W. 2008. Effects of climate change on phenology, frost damage, and floral abundance of montane wildflowers. Ecology 89: 353-362.

Janzen, D.H. 1967. Synchronization of sexual reproduction of trees within the dry season in Central America. Evolution 21: 620-37.

Kovach, W.L. 2003. Oriana - circular statistics for Windows, version 2. Kovach Computing Services, Pentraeth.

Leite, A.V.L. \& Machado, I.C. 2010. Reproductive biology of woody species in Caatinga, a dry forest of northeastern Brazil. Journal of Arid Environments 74: 1374-1380.

Lenza, E. \& Klink, C.A. 2006. Comportamento fenológico de espécies lenhosas em um cerrado sentido restrito de Brasília, DF. Revista Brasileira de Botânica 29: 627-638.

Lenza, E. \& Oliveira, P.E. 2005. Biologia reprodutiva de Tapirira guianensis Aubl. (Anacardiaceae), uma espécie dióica em mata de galeria do Triângulo Mineiro, Brasil. Revista Brasileira de Botânica 28: 179-190.

Madeira, J.A. \& Fernandes G.W. 1999. Reproductive phenology of sympatric species of Chamaecrista (Leguminosae) in Serra do Cipó, Brazil. Journal of Tropical Ecology 15: 463-479.

Martins, A.B.; Goldenberg, R. \& Semir, J. 2009. Flora de Grão-Mogol, Minas Gerais: Melastomataceae. Boletim de Botânica da Universidade de São Paulo 27: 73-96. 
Melo, E. 2000. Polygonaceae da Cadeia do Espinhaço, Brasil. Acta Botanica Brasilica 14: 273-300.

Menezes, N.L. \& Giulietti, A.M. 2000. Campos rupestres. In: Mendonça, M.P. \& Lins, L.V. (eds.). Lista vermelha das espécies ameaçadas de extinção da flora de Minas Gerais. Fundação Biodiversitas, Fundação Zoobotânica de Belo Horizonte, Belo Horizonte. Pp. 65-73.

Miola, D.T.B.; Correia, H.V.L.; Fernandes, G.W. \& Negreiros, D. 2010. Efeito do fogo na fenologia de Syagrus glaucescens Glaz. ex Becc. (Arecaceae). Neotropical Biology and Conservation 5: 146-153.

Miola, D.T.B.; Freitas, C.R.; Barbosa, M. \& Fernandes, G.W. 2011. Modeling the spatial distribution of the endemic and threatened palm shrub Syagrus glaucescens (Arecaceae). Neotropical Biology and Conservation 6: 78-84.

Moeller, D.A. 2004. Facilitative interactions among plants via shared pollinators. Ecology 85: 3289-3301.

Monasterio, M. \& Sarmiento, G. 1976. Phenological strategies of plant species in the tropical savanna and semi-deciduous forest of the Venezuelan Llanos. Journal of Biogeography 3: 325-356.

Moreira, R.G.; McCauley, R.A.; Cortés-Palomec, A.C.; Lovato, M.B.; Fernandes, G.W. \& Oyama, K. 2008. Isolation and characterization of microsatellite loci in Coccoloba cereifera (Polygonaceae), an endangered species endemic to the Serra do Cipó, Brazil. Molecular Ecology Resources 8: 854-856.

Moreira, R.G.; McCauley, R.A.; Cortés-Palomec, A.C.; Lovato, M.B.; Fernandes, G.W. \& Oyama, K. 2010. Spatial genetic structure of Coccoloba cereifera (Polygonaceae), a critically endangered microendemic species of Brazilian rupestrian fields. Conservation Genetics 11: 1247-1255.

Morellato, L.P.C.; Rodrigues, R.R.; Leitão-Filho, H.F. \& Joly, C.A. 1989. Estudo comparativo da fenologia de espécies arbóreas de floresta de altitude e floresta mesófila semidecídua na Serra do Japi, Jundiaí, São Paulo. Revista Brasileira de Botânica 12: 85-98.

Morellato, L.P.C.; Talora, D.C.; Takahasi, A.; Bencke, C.C.; Romera, E.C. \& Zipparro, V.B. 2000. Phenology of Atlantic Rain Forest trees: a comparative study. Biotropica 32: 811-823.

Morellato, L. P. C.; Camargo, M.G.G.; D'Eça Neves, F.F.; Luize, B.G.; Mantovani, A. \& Hudson, I.L. 2010a. The influence of sampling method, sample size, and frequency of observations on plant phenological patterns and interpretation in tropical forest trees. In: Hudson, I.L. \& Keatley, M. (eds.). Phenological research: methods for environmental and climate change analysis. Dordrecht, Springer. Pp. 99-121

Morellato, L.P.C.; Alberti, L.F. \&Hudson, I.L. 2010 b. Applications of circular statistics in plant phenology: a case studies approach. In: Hudson, I.L. \& Keatley, M. (eds.). Phenological research: methods for environmental and climate change analysis. Dordrecht, Springer. Pp. 357-371.

Morellato, L.P.C.; Camargo, M.G.G. \& Gressler, E. 2013. A review of plant phenology in South and Central America. In: Schwartz, M.D. (ed.). Phenology: an integrative environmental science. Dordrecht, Springer. Pp. 91-113.

Munhoz, C.B.R. \& Felfili, J.M. 2005. Fenologia do estrato herbáceo-subarbustivo de uma comunidade campo sujo na Fazenda Água Limpa no Distrito Federal, Brasil. Acta Botanica Brasilica 19: 979-988.

Negreiros, D.; Fernandes, G.W.; Silveira, F.A.O. \& Chalub, C. 2009. Seedling growth and biomass allocation of endemic and threatened shrubs of rupestrian fields. Acta Oecologica 35: 301-310.

Newstrom, L.E.; Frankie, G.W. \& Baker, H.G. 1994. A new classification for plant phenology based on flowering patterns in lowland tropical rain forest trees at La Selva, Costa Rica. Biotropica 26: 141-159.

Oliveira, P.E. 2008. Fenologia e biologia reprodutiva das espécies de cerrado. In: Sano, S.M.; Almeida, S.P. \& Ribeiro, J.F. (eds.). Cerrado: ecologia e flora. EMBRAPA, Planaltina, DF. Pp. 273-287.

Pedroni, F.; Sanchez, M. \& Santos, F.A.M. 2002. Fenologia da copaíba (Copaifera langsdorffii Desf. - Leguminosae, Caesalpinioideae) em uma floresta semidecídua no sudeste do Brasil. Revista Brasileira de Botânica 25: 183-194.

Piedade-Kiill, L.H. \& Ranga, N.T. 2000. Biologia floral e sistema de reprodução de Jacquemontia multiflora (Choisy) Hallier f. (Convolvulaceae). Revista Brasileira de Botânica 23: 37-43.

Pintaud, J.C.; Galeano, G.; Balslev, H.; Bernal, R.; Borchsenius, F.; Ferreira, E.; Granville, J.J.; Mejía, K.; Millán, B.; Moraes, M.; Noblick, L.; Stauffer, F.W. \& Kahn, F. 2008 Las palmeras de América del Sur: diversidad, distribución e historia evolutiva. Revista Peruana de Biología 15: 7-29.

Rathcke, B. \& Lacey, E.P. 1985. Phenological patterns of terrestrial plants. Annual Review of Ecology and Systematics 16: 179-214.

Ribeiro, K.T. \& Fernandes, G.W. 2000. Patterns of abundance of a narrow endemic species in a tropical and infertile montane habitat. Plant Ecology 147: 205-218.

Silva, A.C.; Oliva, M.A.; Vieira, M.F. \& Fernandes, G.W. 2008. Trioecy in Coccoloba cereifera Schwacke (Polygonaceae), a narrow endemic and threatened tropical species. Brazilian Archives of Biology and Technology 51: 1003-1010.

Silveira, F.A.O.; Negreiros, D.; Araújo, L.M. \& Fernandes, G.W. 2012. Does seed germination contribute to ecological breadth and geographic range? A test with 
sympatric Diplusodon (Lythraceae) species from rupestrian fields. Plant Species Biology 27: 170-173.

Staggemeier, V.G. \& Morellato, L.P.C. 2011. Reproductive phenology of coastal plain Atlantic forest vegetation: comparisons from seashore to foothills. International Journal of Biometeorology 55: 843-854.

Tannus, J.L.S.; Assis, M.A. \& Morellato, L.P.C. 2006. Fenologia reprodutiva em campo sujo e campo úmido numa área de Cerrado no sudeste do Brasil, Itirapina - SP. Biota Neotropica 6: 1-23.

Vieira, D.L.M.; Aquino, F.G.; Brito, M.A.; FernandesBulhão, C. \& Henriques, R.P.B. 2002. Síndromes de dispersão de espécies arbustivo-arbóreas em cerrado sensu stricto do Brasil Central e savanas amazônicas Revista Brasileira de Botânica 25: 215-220.

Zar, J.H. 1996. Bioestatistical analysis. Prentice-Hall, New Jersey. 